diversity of both providers and users of health services, every effort should now be made to ensure that assessment is done by a professional who has an understanding of the patient's culture. The authors state that systems are not required to be colour-blind, as this is not helpful. What is required is an acknowledgement of the disadvantages that people from ethnic minorities face and the implementation of measures to address these: denial is not the way forward.

The authors point out the fault lines in the assessment of dangerousness by psychiatrists and the tendency to be more cautious now, thereby leading to overprediction of dangerousness. They argue that this results in greater overprediction in Black people, which in turn is partly responsible for their overrepresentation in secure settings. The suggestion that psychiatry should be explicit about the limitations of making judgements regarding individual dangerousness is welcome, but this should not preclude offering an informed view on the available information and also on the interplay between mental disorder and dangerousness, where indicated. A move away from illness analysis to 'real-life analysis' is advocated, but this view needs more elucidation.

The authors suggest increasing resources to institutions run by Black mental health professionals that aim to provide alternatives to psychiatric hospitals. However, such facilities can at best serve as adjuncts, because many patients, whatever their racial background, need the type of care provided in mainstream psychiatric hospitals, and it is the provision of care in these hospitals that should reflect the diverse cultural and ethnic needs of their users.

The authors argue that appointing more professionals from ethnic minorities in the mental health services does not generally lead to improvement in service provision, because of constraints on those individuals. They note that people previously identified as radical become conservative on being appointed to higher positions. Although therefore questioning the drive to recruit increasing numbers of people from ethnic minorities, the authors also note the relatively small number of Black people in senior positions in various parts of the criminal justice system. Increasing these numbers should strengthen their influence on the changes required for a true multiethnic society. Perhaps a way of assuaging the main concern of the authors is for Black people appointed to senior positions to be made to sign a 'Radicality Act' just like the Official Secrets Act, to ensure that they remain radical after their appointment.

Deji Oyebode Clinical Director of Forensic Mental Health Services, Springfield University Hospital, Shaftesbury Clinic, Glenburnie Road, London SWI7 7DJ, UK

\section{Atypical Antipsychotics}

Edited by B. A. Ellenbroek \& A. R. Cools. Basel: Birkhäuser. 2000. 236 pp.

ISBN $376435948 \times$

This is the best, most concise and comprehensive account of all aspects of atypical antipsychotics that I have seen.

Although atypical antipsychotics have been established as treatments for schizophrenia for nearly 10 years, and the science underpinning their discovery is well described and goes back 30 years, atypicals are the subject of a controversy that frustrates their routine use, particularly in the UK. Consequently, only a minority of patients are on these superior drugs.

Part of the problem is that many different disciplines have a say in this field, from molecular biologists and behavioural psychologists through to clinicians, economists and politicians. Only the most conservative opinions and practices seem to prevail, even in the face of overwhelming evidence for the efficacy of the atypicals.

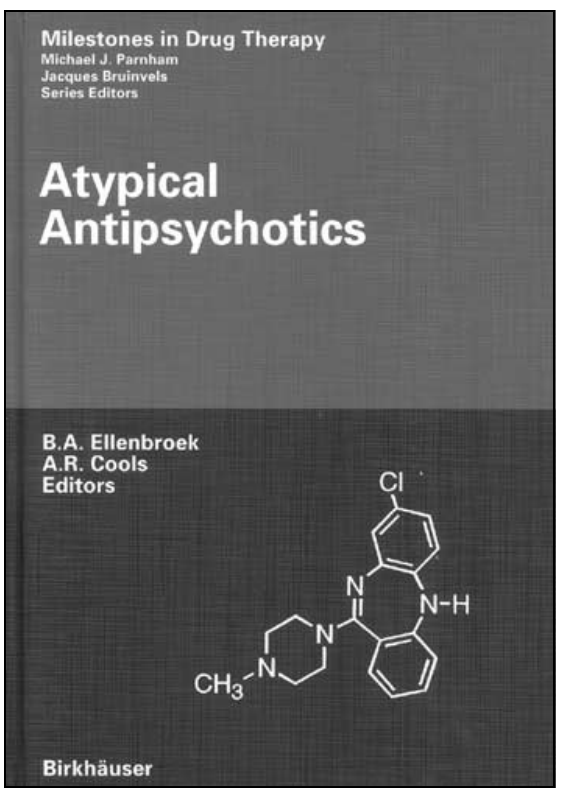

Such is the concern that there is even a review of these agents by the National Institute for Clinical Excellence.

The strength of this volume is that it brings together all of these areas under one cover. There are comprehensive chapters about the drug discovery process, receptor nomenclature and pharmacology, animal models of schizophrenia and imaging. There are also short reviews of each drug that include their clinical characteristics and pharmacological and neurochemical profiles. This is a valuable one-stop source of reference in this field.

The book is not perfect: a multi-review format usually has some overlap as well as variability in quality. Although this collection is the best of its type, some of the reviews are a little one-sided and thinly referenced. However, the majority of chapters are models of clarity and comprehensiveness. I would particularly congratulate Meltzer and Stahl on their contributions.

This is a vital addition to the shelf of every psychopharmacologist and a must for every medical school and research institute library.

Robert Kerwin Professor of Clinical

Neuropharmacology, Division of Psychological

Medicine, Institute of Psychiatry, De Crespigny Park, Denmark Hill, London SE5 8AF, UK

\section{The Psychopharmacologists III}

Interviews by David Healy. London: Arnold 2000. 580 pp. $€ 65.00$ (hb).

ISBN 0340761105

It has been said that scientific progress is made by standing on the shoulders of giants. David Healy must have had a fascinating adventure interviewing the giants that forged psychopharmacology. This is the third volume of his interviews, which have included over 78 contributors, all great or well-known names such as Axelrod and Carlsson (Volume I), Janssen and Schou (Volume II), and Schildkraut, Snyder and Sulser in the present volume.

But did some giants have feet of clay? How is it that the pharmaceutical industry has come to dominate the field? Healy points out that drug companies "are now not simply confined to finding drugs for diseases. They have the power to all but find diseases to suit the drugs they have." Pierre Simon (Sanofi Pharmaceuticals) remarks: "In the 